\title{
ORIGINAL RESEARCH \\ MR Imaging of Adenomas of the Nonpigmented Ciliary Epithelium of the Eye
}

J. Xian

Q. Xu

Z. Wang

B. Yang

W. Wei

Z. Yan

J. Sun

J. Shi

G.S. Young

BACKGROUND AND PURPOSE: ANPCEs are rare benign tumors of the eye arising from the NPCE in adults, which may be clinically mistaken for melanoma. This study was undertaken to delineate clinical and MR imaging features of these tumors.

MATERIALS AND METHODS: Clinical presentation and MR imaging findings of 8 patients ( 6 women and 2 men; median age, 51 years) with pathologically confirmed ANPCEs were retrospectively reviewed. Location, size, shape, margin, signal intensity, and gadolinium-enhancement characteristics of all tumors were evaluated. Signal intensity and degree of enhancement were graded in comparison with the ipsilateral lacrimal gland.

RESULTS: MR imaging revealed a circumscribed enhancing mass within the ciliary body of the eye in all 8 patients. The mass was ovoid in 6 patients and spheric in 2. Gadolinium enhancement was marked in 4 lesions and moderate in the other 4 . Both T1 and T2 relaxation times were qualitatively identical to those in the lacrimal gland in 2 tumors. In the remaining 6 tumors, the T1 was identical to and the T2 longer than that in the lacrimal gland.

CONCLUSIONS: ANPCE should be included in the differential diagnosis of a spheric or ovoid enhancing ciliary body mass with T1 similar to that in the lacrimal gland and T2 equal to or longer than that in the lacrimal gland.

ABBREVIATIONS: ANPCE $=$ adenoma of the nonpigmented ciliary body epithelium; FSE $=$ fast spin-echo; Hyper = hyperintense; Hypo = hypointense; Inflammation = intra-operative or pathologic evidence of inflammation; I = inferior; IOP = intraocular pressure; Iso = isointense; IT = inferotemporal; $\mathrm{L}=$ left; $\mathrm{N}=$ normal; NPCE = nonpigmented ciliary body epithelium; $\mathrm{R}=$ right; Resection = local tumor resection; $\mathrm{S}=$ superior; $\mathrm{SE}=$ spin-echo; $\mathrm{SN}=$ superonasal; $\mathrm{ST}=$ superotemporal; SV = sentinel vessel (prominently dilated episcleral blood vessels overlying the tumor); $\mathrm{T} 1 \mathrm{WI}=\mathrm{T} 1$-weighted imaging; $\mathrm{T} 2 \mathrm{WI}=\mathrm{T} 2$-weighted imaging

A NPCEs are rare benign tumors of unknown pathogenesis arising with equal frequency in adults of both sexes within the posterior chamber of the anterior cavity of the globe. ${ }^{1}$ Because they arise from the epithelial covering of the posterior aspect of the ciliary body, ANPCEs more commonly protrude posteriorly through the posterior chamber and into the vitreous chamber of the globe but may alternatively extend anteriorly through the peripheral iris into the anterior chamber. ${ }^{2-7}$ Although ANPCE is often asymptomatic, it may produce local inflammation, mass effect, or infiltration of the adjacent lens, resulting in development of a secondary cataract or lens subluxation, either of which can cause the patient to present with vision loss. ${ }^{1-4,6}$ Because these tumors are located behind the

\section{Received July 13, 2009; accepted after revision September 19}

From the Departments of Radiology (J.X., O.X., Z.W., B.Y., Z.Y., J.Sun) and Ophthalmology (W.W., J.Shi), Capital Medical University Beijing Tongren Hospital, Beijing, China; and Department of Radiology (G.S.Y.), Brigham and Women's Hospital, Harvard Medical School, Boston, Massachusetts.

Junfang Xian and Qinggang $\mathrm{Xu}$ shared equally in the authorship of this article and are co-first authors

This work was in part supported by a grant (2004-B-31) from New Star of Beijing Science and Technology Committee, China; and grants $(7062019,7082026)$ from Beijing Nature and Science, China

Please address correspondence to Junfang Xian, MD, Department of Radiology, Capital Medical University Beijing Tongren Hospital, Beijing, China; e-mail: cjr.xianjunfang@ vip.163.com; or Geoffrey S. Young, MD, Department of Radiology, Brigham and Women's Hospital, Harvard Medical School, Boston, MA 02115; e-mail: gsyoung@partners.org

Indicates open access to non-subscribers at www.ajnr.org

DOI 10.3174/ajnr.A1947 iris in the ciliary body, they are difficult to see on slit-lamp examination.

Although the preferred treatment for ANPCEs is local resection sparing the globe, they are often clinically indistinguishable from the more common ciliary body malignant melanoma that arises in the same location and thus are sometimes treated with unnecessary enucleation. ${ }^{2-3,5-10}$ Local resection or incisional biopsy followed by a second surgery for more extensive definitive resection, if needed, after pathologic diagnosis may be a desirable alternative if a benign lesion such as an ANPCE is suspected. Unfortunately, this is undesirable for patients with suspected melanoma because the 2 surgeries that are needed to achieve definitive resection result in increased expense and increased risk of local and distant metastatic spread. Because melanoma is much more common, it is not practical or desirable to adopt this approach in all cases. This problem provides strong motivation for researchers seeking to use preoperative MR imaging to define a limited subset of patients with a high likelihood of ANPCE. If ANPCE can confidently be included high in the preoperative differential diagnosis, such patients could be managed more conservatively. This change would decrease the number of unnecessary enucleations.

Because ANPCE lacks the melanin that confers characteristically short T1 and T2 relaxation times on most ocular melanomas, it may be possible to suggest this diagnosis in many cases on the basis of preoperative MR imaging. ${ }^{11-14}$ The most recent and detailed review of the MR imaging of ANPCE literature revealed 4 published cases. ${ }^{15}$ Of these 4 cases, 3 tumors 
Table 1: Patient clinical data

\begin{tabular}{|c|c|c|c|c|c|c|c|c|c|c|c|}
\hline $\begin{array}{l}\text { Case } \\
\text { No. } \\
\end{array}$ & Age & Sex & Eye & Prior Trauma & Vision Loss & IOP & SV & Cataract & Subluxed Lens & Inflammation & Management \\
\hline 1 & 45 & $\mathrm{~F}$ & $\mathrm{~L}$ & No & Yes & $\mathrm{N}$ & No & Yes & Yes & Yes & Resection \\
\hline 2 & 53 & M & L & No & Yes & $\mathrm{N}$ & Yes & Yes & Yes & Yes & Enucleation \\
\hline 3 & 53 & $\mathrm{~F}$ & L & No & Yes & $\mathrm{N}$ & Yes & Yes & Yes & Yes & Resection \\
\hline 4 & 53 & $\mathrm{~F}$ & $\mathrm{R}$ & No & Yes & $\mathrm{N}$ & Yes & Yes & Yes & No & Resection \\
\hline 5 & 35 & $\mathrm{~F}$ & $\mathrm{R}$ & No & Yes & $\mathrm{N}$ & Yes & Yes & Yes & No & Resection \\
\hline 6 & 49 & $\mathrm{~F}$ & L & No & Yes & $\mathrm{N}$ & Yes & Yes & No & No & Resection \\
\hline 7 & 67 & M & $\mathrm{R}$ & No & Yes & $\mathrm{N}$ & Yes & No & Yes & No & Enucleation \\
\hline 8 & 19 & $\mathrm{~F}$ & $\mathrm{R}$ & No & No & $\mathrm{N}$ & No & No & No & No & Resection \\
\hline
\end{tabular}

had either a T1 or T2 similar to that in the vitreous and thus atypical for melanoma, supporting the hypothesis that the T1 and T2 of ANPCE might allow differentiation from adenoma in some cases. However, 2 of the 4 lesions had T1 shorter than that of the vitreous (like melanoma), 2 had T2 shorter than that of the vitreous (like melanoma), and 1 of the lesions had T1 and T2 shorter than those of the vitreous and was thus indistinguishable from melanoma. ${ }^{6,15}$ The variability of reported findings of $\mathrm{T} 1$ and $\mathrm{T} 2$ relative to the vitreous and the overlap of these findings with the typical imaging features of melanoma motivated us to design a retrospective series to better delineate the clinical and MR imaging features of ANPCE in a larger series and, if possible, to identify MR imaging markers worthy of investigation in a future differential diagnostic study.

\section{Materials and Methods}

An electronic medical record review, approved by our institutional review board, revealed 8 patients who presented with pathologically confirmed ANPCE between June 2004 and January 2009, including 6 women and 2 men whose ages at the time of diagnosis ranged from 19 to 67 years (median, 51 years) (Table 1). Patient data extracted from the medical records included age, sex, involved eye; history of ocular trauma or inflammation, visual acuity, and intraocular pressures were recorded. Tumor location, size, and color were noted. The presence or absence of prominently dilated episcleral sentinel blood vessels, cataracts, subluxation of the lens, secondary inflammatory signs, and extraocular extension was noted. Type of treatment was recorded.

Available MR imaging data were retrieved for all 8 patients. All patients had undergone orbit MR imaging with dual 3-inch $(7.62 \mathrm{~cm})$ surface coils, performed on a 1.5T clinical MR imaging scanner (Signa TwinSpeed; GE Healthcare, Milwaukee, Wisconsin). Motion artifacts were reduced by instructing the patients to keep their eyes shut and to open and close them several times during the measurement breaks. The standard institutional orbit imaging protocol used in all cases included high-resolution SE T1WI and FSE T2WI with 3- to 4-mm spacing, 0.3 - to 0.5 - $\mathrm{mm}$ sections, $100 \times 100 \mathrm{~mm}$ FOV, and $288 \times 224$ matrix. SE T1WI parameters were the following: TR/TE, 600/11.1 ms, 2 excitations, 1:39 acquisition time. FSE T2WI acquisition parameters were the following: TR/TE, 3000/120 ms, 3 excitations, and 1:36 acquisition time. Pregadolinium T1WIs and T2WIs in 2 planes (axial plus coronal or sagittal) and frequency-selective fat-suppressed axial postgadolinium T1WIs were acquired in all cases. Gadopentetate dimeglumine $(0.1 \mathrm{mmol} / \mathrm{kg}$, Magnevist; Bayer Schering Pharma, Berlin, Germany) was injected at a rate of $2.0 \mathrm{~mL} / \mathrm{s}$ through a 21 -gauge intravenous line with a power injector.

MR imaging findings of the tumor were evaluated with emphasis on the location, size, shape, signal intensity, and enhancement. The signal intensity of the tumor was evaluated in comparison with the lacrimal gland and the vitreous. Signal intensity on postgadolinium T1WI higher than that of the lacrimal gland was defined as marked enhancement, and the signal intensity equal to that of the lacrimal gland was defined as moderate enhancement.

\section{Results}

Table 1 provides clinical detail on the 8 patients. No history of trauma was reported for any patient. No secondary glaucoma was identified. Slit-lamp examination revealed secondary cataract ipsilateral to the tumor in 6 patients, including 3 focal and 3 total cataracts. Prominent dilated episcleral blood vessels (sentinel vessels) overlying the tumor were detected in 6 patients by visual examination. In 6 cases, the tumor appeared nonpigmented with slit-lamp observation but was revealed to be white to light-tan during gross pathology. In the remaining 2 patients, the tumor appeared melanotic by slit-lamp observation but again proved to be amelanotic under gross pathologic examination. Intraocular inflammation was identified in 3 patients during surgery. Corresponding inflammatory cell infiltrate with lymphocytes and plasma cells and evidence of chronic inflammation were observed pathologically.

Table 2 details the MR imaging features of all 8 patients. MR imaging revealed a slightly irregular margin in 2 patients (Fig 1) and a smooth margin in 6. In 2 patients, MR imaging revealed tumor involvement of the iris and extension into the anterior chamber of the globe through the peripheral iris (Fig 2 ). This extension into the anterior chamber was only seen in patients with a displaced lens. The tumor ranged from 3 to 8 $\mathrm{mm}$ (median, $5 \mathrm{~mm}$ ) in diameter. No extrascleral extension of tumor was detected in any case.

The 6 patients with tumors that appeared nonmelanotic under slit lamp were managed by local tumor-only resection. The 2 patients with tumors that appeared melanotic under slit lamp had been referred for enucleation of a suspected melanoma and declined the surgeon's suggestion of local tumor excision. Although the pathology literature has also reported that ANPCEs have an irregular surface, ${ }^{2,3,6}$ only slight irregularity was seen in our series, and this, in only 2 patients. In the remaining 6 patients in our study, the tumor surface was smooth on pathology, better corresponding to the MR imaging findings than to the previous reports.

\section{Discussion}

ANPCEs are rare slow-growing benign tumors arising from the inner surface of the ciliary body of the eye. Tumors of the NPCE can be divided into congenital and acquired tumors. The best known congenital tumor, medulloepithelioma, arises 


\begin{tabular}{|c|c|c|c|c|c|c|c|c|c|}
\hline \multirow[b]{3}{*}{ Case No. } & \multirow[b]{3}{*}{ Quadrant } & \multirow[b]{3}{*}{ Shape } & \multirow[b]{3}{*}{ Subluxed Lens } & \multirow[b]{3}{*}{ Size $(\mathrm{mm})$} & \multicolumn{4}{|c|}{ Signal Intensity Relative to } & \multirow[b]{3}{*}{ Enhancement } \\
\hline & & & & & \multicolumn{2}{|c|}{ Lacrimal Gland } & \multicolumn{2}{|c|}{ Vitreous } & \\
\hline & & & & & T1WI & $\mathrm{T} 2 \mathrm{WI}$ & T1WI & $\mathrm{T} 2 \mathrm{WI}$ & \\
\hline 1 & ST & Oval & Yes & $6 \times 6 \times 4$ & Iso & Hyper & Hyper & Нyро & Marked \\
\hline 2 & S & Oval & Yes & $6 \times 6 \times 5$ & Iso & Iso & Hyper & Нуро & Moderate \\
\hline 3 & IT & Oval & Yes & $5 \times 4 \times 4$ & Iso & Hyper & Hyper & Iso & Marked \\
\hline 4 & I & Round & Yes & $5 \times 5 \times 5$ & Iso & Iso & Hyper & Нуро & Moderate \\
\hline 5 & ST & Oval & Yes & $4 \times 3 \times 3$ & Iso & Hyper & Hyper & Нуро & Marked \\
\hline 6 & । & Round & No & $3 \times 3 \times 3$ & Iso & Hyper & Hyper & Iso & Moderate \\
\hline 7 & SN & Oval & Yes & $6 \times 5 \times 4$ & Iso & Hyper & Hyper & Нypo & Moderate \\
\hline 8 & IT & Oval & No & $8 \times 7 \times 4$ & Iso & Hyper & Iso & Нуро & Marked \\
\hline
\end{tabular}
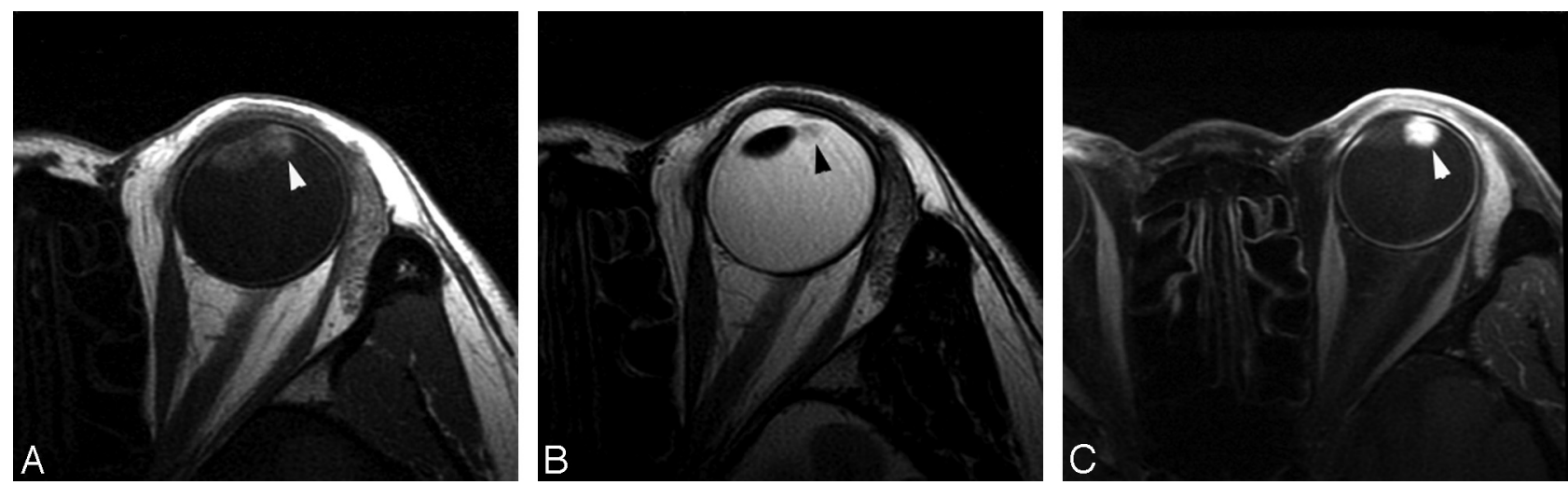

Fig 1. Case 1. ANPCE in the left eye in a 45-year-old woman. Compared with the lacrimal gland tissue, the tumor (arrowhead) demonstrates isointensity on T1WI (A), hyperintensity on FSE T2WI $(B)$, and marked enhancement on postcontrast T1WI $(C)$. The tumor has a slightly irregular surface. Displacement of the lens is seen.
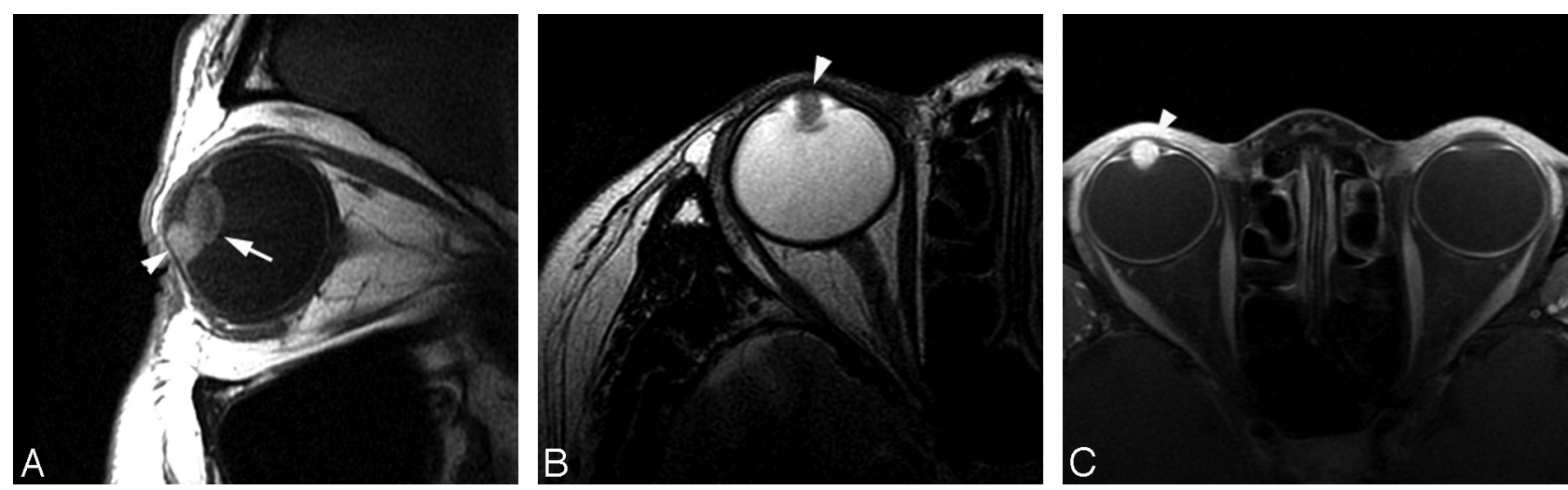

Fig 2. Case 4. ANPCE in the right eye in a 53-year-old woman. With respect to the lacrimal gland tissue, the tumor (arrowhead) shows isointensity on obliquely sagittal T1WI (A) and axial FSE T2WI $(B)$ and moderate enhancement on axial postcontrast T1WI $(C)$. Displacement of the lens (arrow, $A$ ) is identified. The iris is involved.

in the embryonic or early postnatal period. ${ }^{1,16}$ Acquired adenoma and adenocarcinoma of the NPCE are less common and arise primarily in middle age (mean age, 45 years) in patients of both sexes. ${ }^{2,3,6}$ The pathogenesis of ANPCE remains unknown. Although some authors speculate that these tumors might represent a nodular reactive proliferation initiated by trauma or inflammation, ${ }^{6}$ most published cases report no history of trauma or severe prior intraocular inflammation, ${ }^{2-10}$ and none was elicited in our patients. Although ANPCEs are benign, they can exhibit locally aggressive behavior, resulting in cataract ipsilateral to the tumor. ${ }^{1}$ For this reason, patients with ANPCE may present with painless vision loss due to secondary focal cataracts developing at an early stage or total cataracts or subluxed lens developing later. ${ }^{2-4,6}$ Similarly, although generally taken to be signs of intraocular malignancy found in malignant melanoma or metastases, both intraocular neovascularization and development of episcleral sentinel vessels overlying the tumor may arise in patients with ANPCE, sometimes leading to cystoid macular edema. ${ }^{2,6,10}$

The 2 tumors in our study that appeared melanotic under slit-lamp observation proved to be amelanotic by gross pathologic examination, consistent with the prior pathology literature. $^{2,3,6}$ In retrospect, it seems likely that in these 2 cases, pigmented epithelium adjacent to the tumor was misinterpreted as a melanotic surface of the tumor by slit-lamp observation. These 2 patients were referred for and underwent enucleation of the eyes that might have been spared had ANPCE been suspected preoperatively. This experience emphasizes both the present need to better define the appearance of and increase physician awareness of ANPCE within the differential 
diagnosis of intraocular masses. It also suggests a clear need for continuing investigation of complementary noninvasive imaging techniques, including MR imaging methods that could differentiate ANPCE from the more common malignant intraocular melanoma and metastases.

Because the literature to date neither describes ANPCE MR imaging findings in detail nor presents any indication of how MR imaging could contribute to the differential diagnosis of ANPCE from melanoma or other malignant lesions, ${ }^{6,15}$ we set out primarily to define in detail the conventional MR imaging of ANPCE with a view to identifying features distinct enough to merit investigation in future diagnostic controlled trials. Because the prior literature reporting the T1 of ANPCE, uveal melanoma, and metastasis in comparison with the vitreous has demonstrated a broad overlap between these lesions, ${ }^{17}$ we evaluated the T1, T2, and degree of enhancement of the ANPCE with reference to the lacrimal gland as well as the vitreous.

On pregadolinium imaging, all 8 ANPCEs were found to have a T1 similar to that of the lacrimal gland, including the 2 cases clinically misdiagnosed as melanotic on slit-lamp examination. Although we did not design the current study to include formal assessment of uveal melanoma and we have not found any literature directly reporting the T1 of melanoma with respect to lacrimal gland, in our experience, the T1 of malignant melanoma is generally shorter than that in the lacrimal gland, suggesting that this feature may deserve further study as a potential differentiator of ANPCE in a future controlled trial. As expected from the fact that the T1 of the vitreous is longer than that of the normal lacrimal gland, in 7 of the 8 cases, the ANPCE T1 was also shorter (higher signal intensity on T1WI) than that in the vitreous. In the remaining case, the ANPCE T1 was essentially identical to that of the vitreous. Because nearly all intraocular melanoma and metastases have a T1 shorter than that in the vitreous, comparison of tumor with vitreous on T1WI is unlikely to contribute to the differential diagnosis of these lesions.

On T2WI, 6 of our ANPCEs had T2s longer than those in the lacrimal gland (producing higher signal intensity on T2WI), and 2 had T2 similar to that of the lacrimal gland. Again, because we recognize that the design of our current study does not include formal assessment of uveal melanoma, in our experience, the T2 of malignant melanoma is generally shorter than that of the lacrimal gland, suggesting that T2 longer than or equal to that of the lacrimal gland could contribute to the differentiation of ANPCE from melanoma and that this feature deserves to be included in further differential diagnostic MR imaging studies.

The T2 of ANPCE in our study was also shorter than that of the vitreous in most cases. Unfortunately, the literature documents that melanoma characteristically also has a T2 shorter than that of the vitreous, suggesting that lacrimal gland tissue may offer a superior reference to the vitreous or orbital fat for assessing the signal intensity of ciliary body tumors on T2WI and T1WI.

Our results indicate that ANPCE most commonly presents as an oval or round mass. While roughly one-third of melanomas have a characteristic mushroom-shape not observed in our series, up to two-thirds of melanomas and nearly half of metastases may appear as oval or round masses. ${ }^{11,12,14,17,18}$
Thus, while a mushroom shape may be used to favor melanoma over ANPCE, an oval or round shape cannot reliably differentiate ANPCE from melanoma or metastasis. In addition, MR imaging evidence of extraocular growth was not seen in our series and would be very unusual for a benign lesion such as ANPCE but has frequently been reported in malignant melanoma. ${ }^{11,12,14}$ Thus, although the round or oval shape and the absence of extraocular extension characteristically observed in ANPCE cannot be used to favor ANPCE over melanoma to any significant degree, the observation of a mushroom shape, or more conclusively extraocular extension, may help to exclude ANPCE from the differential diagnosis.

The most important consideration in the differential diagnosis of tumors of the ANPCE is ciliary body melanoma because melanoma is more common and, unlike ANPCE, requires enucleation. Other main considerations in the differential diagnosis are medulloepithelioma, adenoma, or adenocarcinoma of the ciliary pigment epithelium, leiomyoma, schwannoma, metastatic carcinoma, and granuloma. ${ }^{2-6,12-14,16,19,20}$

Unlike ANPCE, many of these lesions require enucleation. Medulloepithelioma, a congenital tumor with its onset in the first decade of life and often associated with lens coloboma, iris neovascularization, and signs of persistent primary vitreous, ${ }^{16}$ can be differentiated readily by clinical findings. Metastatic carcinoma is more likely to occur in patients with a history of carcinoma and concurrent metastases elsewhere. ${ }^{12,13}$ Ciliary body granuloma is always associated with more severe uveal inflammation, and the patient tends to have systemic manifestations of a granulomatous condition. ${ }^{12,13}$

Two relatively less common lesions in the differential diagnosis that may present difficulty clinically include adenomas or adenocarcinomas of the ciliary pigment epithelium. The distinction between these 2 entities is based on the degree of histologic invasion, but because these may be distinguished from unpigmented lesions such as ANPCE on slit-lamp examination due to their pigmented appearance, they could be confused with melanoma. Our limited experience suggests that the 2 adenomas of the ciliary pigment epithelium we have encountered were hyperintense on T1WIs and hypointense on T2WIs relative to lacrimal gland tissue and presented a potential clinical and MR imaging mimic of melanoma. Similarly, leiomyoma and schwannoma of ciliary body may be indistinguishable from ANPCE by clinical or imaging findings but may also be treated with local resection, like ANPCE. ${ }^{12,20}$ The most appropriate management for ANPCE is generally local tumor resection only, rather than enucleation, if ANPCE is detected clinically at a relatively early stage.

Although ANPCE can behave aggressively locally, it appears less likely to recur after removal of the tumor locally. The life expectancy for patients with ANPCE is excellent. Although ANPCE may evolve into adenocarcinomas of the NPCE, adenocarcinomas of the NPCE have no tendency to metastasize, so differentiation of these 2 pathologically distinct but clinically similar entities is of little practical importance. ${ }^{6}$ Thus while a method to suspect ANPCE rather than melanoma preoperatively in appropriate cases would not completely resolve the problem of diagnosing ocular masses, this differentiation could prevent the unnecessary enucleation of a number of 
benign lesions and seems an important goal for orbital MR imaging.

\section{Conclusions}

Adenoma of the NPCE is an important locally resectable benign entity that should be included in the differential diagnosis of ocular masses when an oval or round mass with a T1 similar to that in the lacrimal gland and a T2 longer or equal to that in the lacrimal gland is detected on MR imaging in the ciliary body. Because these tumors may be mistaken clinically for the more common uveal melanoma, which is treated with enucleation, a diagnostic controlled study seems indicated to assess whether these MR imaging characteristics can be used to distinguish ANPCE from melanoma and other ocular masses.

\section{References}

1. Mansoor S, Qureshi A. Ciliary body adenoma of non-pigmented epithelium. J Clin Pathol 2004;57:997-98

2. Appolloni R, Modesti M, Pecorella I, et al. Uncommon cause of juvenile cataract: adenoma of the nonpigmented ciliary epithelium. J Cataract Refract Surg 2008;34:1997-2001

3. Elizalde J, Ubia S, Barraquer RI. Adenoma of the nonpigmented ciliary epithelium. Eur J Ophthalmol 2006;16:630-33

4. Murphy MF, Johnston PB, Lyness RW. Adenoma of the non-pigmented epithelium of the ciliary body. Eye 1997;11:419-20

5. Cursiefen C, Schlötzer-Schrehardt U, Holbach LM, et al. Adenoma of the nonpigmented ciliary epithelium mimicking a malignant melanoma of the iris. Arch Ophthalmol 1999;117:113-16

6. Shields JA, Eagle RC Jr, Shields CL, et al. Acquired neoplasms of the nonpigmented ciliary epithelium (adenoma and adenocarcinoma). Ophthalmology 1996;103:2007-16
7. Shields JA, Augsburger JJ, Waller $\mathrm{PH}$, et al. Adenoma of the nonpigmented epithelium of the ciliary body. Ophthalmology 1983;90:1528-30

8. McGowan HD, Simpson ER, Hunter WS, et al. Adenoma of the nonpigmented epithelium of the ciliary body. Can J Ophthalmol 1991;26:328-33

9. Grossniklaus HE, Lim JI. Adenoma of the nonpigmented ciliary epithelium. Retina 1994;14:452-56

10. Suzuki J, Goto H, Usui M. Adenoma arising from nonpigmented ciliary epithelium concomitant with neovascularization of the optic disk and cystoid macular edema. Am J Ophthalmol 2005;139:188-90

11. De Potter P, Flanders AE, Shields JA, et al. The role of fat-suppression technique and gadopentetate dimeglumine in magnetic resonance imaging evaluation of intraocular tumors and simulating lesions. Arch Ophthalmol $1994 ; 112: 340-48$

12. Mafee MF. Uveal melanoma, choroidal hemangioma, and simulating lesions: role of MR imaging. Radiol Clin North Am 1998;36:1083-99

13. Mafee MF. The eye. In: Som PM, Curtin HD, eds. Head and Neck Imaging. 4th ed. St Louis: Mosby; 2003:508-16

14. Lemke AJ, Hosten N, Bornfeld N, et al. Uveal melanoma: correlation of histopathologic and radiologic findings by using thin-section MR imaging with a surface coil. Radiology 1999;210:775-83

15. Nakazawa T, Abe T, Sato Y, et al. Magnetic resonance imaging of a non-pigmented adenoma of the ciliary epithelium. Acta Ophthalmol Scand 2000;78: $470-73$

16. Shields JA, Eagle RC Jr, Shields CL, et al. Congenital neoplasms of the nonpigmented ciliary epithelium (medulloepithelioma). Ophthalmology 1996;103: 1998-2006

17. Lemke AJ, Hosten N, Wiegel T, et al. Intraocular metastases: differential diagnosis from uveal melanomas with high-resolution MRI using a surface coil. Eur Radiol 2001;11:2593-601

18. Mafee MF, Peyman GA, Grisolano JE, et al. Malignant uveal melanoma and simulating lesions: MR imaging evaluation. Radiology 1986;160:773-80

19. Shields JA, Shields CL, Gündüz K, et al. Adenoma of the ciliary body pigment epithelium: the 1998 Albert Ruedemann, Sr, memorial lecture, Part 1. Arch Ophthalmol 1999;117:592-97

20. Xian J, Xu X, Wang Z, et al. Magnetic resonance imaging findings of the uveal schwannoma. AJNR Am J Neuroradiol 2009;30:769-73 